\section{Preferential Growth of Metallic SWNTs Achieved}

One obstacle to the implementation of single-walled carbon nanotubes (SWNTs) in electronic devices is that their synthesis results in a mixture of conducting and semiconducting species. Progress has been made in separating SWNTs based on their conductivities, which is determined by chirality. Some progress has been made in controlling SWNT structure during growth (e.g., semiconducting SWNTs are produced preferentially by plasma-enhanced chemical vapor deposition), demonstrating that somewhat different mechanisms lead to different chiralities. Recently, A.R. Harutyunyan and co-researchers at the Honda Research Institute, Columbus, Ohio, in collaboration with T.M. Paronyan of the University of Louisville, S.M. Kim of Purdue University, and their colleagues, were able to increase the fraction of CNTs with metallic conductivity grown from Fe nanocatalysts from $33 \%$ to a maximum of $91 \%$ by varying the conditions under which the catalyst was annealed.

As reported in the October 2 issue of Science (DOI: 10.1126/science.1177599; p. 116), Harutyunyan and co-researchers annealed in situ Fe nanocatalysts deposited onto a flat $\mathrm{SiO}_{2} / \mathrm{Si}$ support, using an ambient atmosphere of $\mathrm{He}$ or $\mathrm{Ar}$, and varying ratios of $\mathrm{H}_{2}$ and $\mathrm{H}_{2} \mathrm{O}$. Using methane as the carbon source, SWNT synthesis was performed at $860^{\circ} \mathrm{C}$. The researchers observed with scanning electron microscopy that increasing the concentration of reductive species (Ar: $\mathrm{H}_{2}$ was increased from 9:1 to 8:2 at 840 Torr with 3.5 mTorr $\mathrm{H}_{2} \mathrm{O}$ ) during catalyst conditioning resulted in higher densities of SWNTs on the substrate. This led them to perform a systematic study of SWNT growth on catalysts annealed in situ under varying ambient conditions. The ratio of metallic to semiconducting tubes was obtained by measuring the SWNT's Raman breathing modes, specifically, $R=I_{\text {met }} / I_{\text {sem }}$, where $I_{\text {met }}$ and $I_{\text {sem }}$ are the integrated intensities of the metallic and semiconducting SWNTs, respectively. The researchers observed an increase in $R$ by (1) replacing $\mathrm{Ar}$ with $\mathrm{He}$; (2) increasing the $\mathrm{H}_{2}$ content in the Ar- $\mathrm{H}_{2}$ atmosphere; and (3) increasing the annealing time from 1 minute to 10 minutes. A very high $R$ value of 20.2, which corresponds to over $90 \%$ metallic SWNTS, was obtained with a $\mathrm{He}: \mathrm{H}_{2}$ ratio of $8: 2$ and an annealing duration of 10 minutes.

The researchers verified their Ramanspectra analysis by measuring field-effect transistor performance for 47 individual SWNTs and characterizing them according to their source-drain current. Another set of experiments demonstrated that the catalyst annealed in the presence of $\mathrm{H}_{2} \mathrm{O}$ in the ambient atmosphere together with He promotes the growth of metallic tubes whereas semiconducting tubes are favored by catalysts annealed with an ambient atmosphere of $\mathrm{H}_{2} \mathrm{O}$ and Ar. The researchers also investigated the mechanism of tube formation with in situ transmission electron microscopy of the Fe nanocatalysts under varying gaseous environments in analogy with the SWNTsynthesis conditions. Differences in both morphology and coarsening behavior of the nanocatalyst were observed.

The researchers said that "these catalyst rearrangements demonstrate that there are correlations between catalyst morphology and resulting nanotube electronic structure and indicate that chiralselective growth may be possible."

STEVEN TROHALAKI

\section{Quasicrystalline Order Revealed in Nanoparticle Superlattices}

Quasicrystals are a class of materials that show sharp diffraction peaks despite presenting forbidden symmetry operations in classical crystallography. D.V. Talapin and M.I. Bodnarchuk from the University of Chicago; E.V. Shevchenko from Argonne National Laboratory; and X. Ye, J. Chen, and C.B. Murray from the University of Pennsylvania, have reported in the October 15 issue of Nature (DOI: 10.1038 /nature08439; p. 964) that different binary nanoparticle colloidal systems can self-assemble into 12-fold rotational quasicrystalline order. According to the researchers, the compositional flexibility demonstrated that quasicrystal ordering could be a relatively common phenomenon in nanocrystal solids, with suitable size ratios between particles.

\section{Addendum}

David N. Seidman and Krystyna Stiller, Guest Editors of the theme "A Renaissance in Atom-Probe Tomography" (MRS Bulletin, October 2009), note that in 1973, J.A. Panitz invented the $10-\mathrm{cm}$ atom probe (discussed on p. 745) - now called the imaging atom probe-which is the progenitor of all atomprobe tomographs, citing the following references: J.A. Panitz, Rev. Sci. Instrum. 44, 1034 (1973) and J.A. Panitz, "Field Desorption Spectrometer,” U.S. Patent 3868507 (1975).
}

The researchers obtained the quasicrystalline nanoparticle assemblies by evaporating relatively concentrated colloidal solutions of 13.4-nm $\mathrm{Fe}_{2} \mathrm{O}_{3}$ and 5.0-nm Au monodisperse nanoparticles capped with oleic acid and dodecanethiol molecules, respectively, in tetracloroethylene at $50^{\circ} \mathrm{C}$ under reduced pressure $(\sim 3.2 \mathrm{kPa})$ on a carbon-coated transmission electron microscopy grid or a silicon nitride membrane tilted by $60^{\circ}$ or $70^{\circ}$. The researchers used the surfactant molecules to introduce short-range steric repulsion that counterbalanced the van der Waals forces and prevented uncontrollable aggregation of nanocrystals in the colloidal solution. They observed that during this process the nanocrystals self-assembled in $\mathrm{AlB}_{2^{-}}$and $\mathrm{CaB}_{6}$-type phases, and on the $\left(3^{2} .4 .3 .4\right)$ Archimedean tiling structure, depending on the $\mathrm{Fe}_{2} \mathrm{O}_{3}$-to-Au nanoparticle ratio. This structure is formed by five planar polygons (three triangles and two squares) sharing a common vertex in such a way that they fill the plane with no overlaps and no gaps, depending on the Fe. The nomenclature of these structures lists in order the polygons that meet at each vertex using integers that correspond to the numbers of sides of the polygons. So, the $\left(3^{2} .4 .3 .4\right)$ structure consists of two triangles sharing a common edge, surrounded by two squares at each side of the triangles, and filling the empty space by another triangle, all of them sharing a common vertex. In proximity to the ( $3^{2}$.4.3.4) phase in the binary phase diagram of these nanoparticles, the researchers observed reproducible formation of a type of binary superstructure without translational symmetry. They observed that these structures showed sharp electron diffraction patterns revealing dodecagonal rotational symmetry, a symmetry operation forbidden in periodic structures. The researchers identified these self-assembled nanoparticle superstructures as dodecagonal quasicrystals (DDQC), a phase that formed also from colloidal solutions containing 12.6-nm $\mathrm{Fe}_{3} \mathrm{O}_{4}$ and 4.7-nm Au nanocrystals, and 9-nm PbS and 3-nm Pd nanocrystals, with size ratios between particles of $\sim 0.43$.

The researchers said that the space-filling factor had a significant effect on the relative stabilities of binary nanoparticle phases, and the quasi-periodicity could be a result of maximizing the entropy of arrangement of square and triangular "tiles." They consider that the discontinuity in the entropy density corresponding to the DDQC state might provide a mechanism for locking the quasicrystalline state over a range of nanocrystal concentration ratios. The researchers think that these studies will provide insight into the formation of the quasicrystal phase in atomic systems, and can be used as a convenient platform for detailed investigation of quasicrystal properties.

JOAN J. CARVAJAL 